\title{
Technical Implications of Neglecting Compositional Grading Effects in Petroleum Reservoir Simulation Models
}

Ikechi Igwe , *Jebraeel Gholinezhad ${ }^{+}$, Mohamed Galal Hassan Sayed ${ }^{++}$, Frank Ogbuagu $^{+++}$

${ }^{+}$School of Energy and Electronic Engineering, Anglesea Building, University of Portsmouth, Portsmouth, PO1 3DJ, United Kingdom.

${ }^{++}$Chemical Engineering Building 30, University of Southampton, Southampton SO17 IBJ, United Kingdom.

${ }^{+++}$Chevron Nigeria Limited, 2 Chevron Drive, Lekki, Lagos State, Nigeria

\section{SUPPORTING INFORMATION}

- Probability Distribution Curves for Oil Originally In-place (OOIP) and Gas Originally In-place (GOIP)for the Various Initialized Reservoir Models

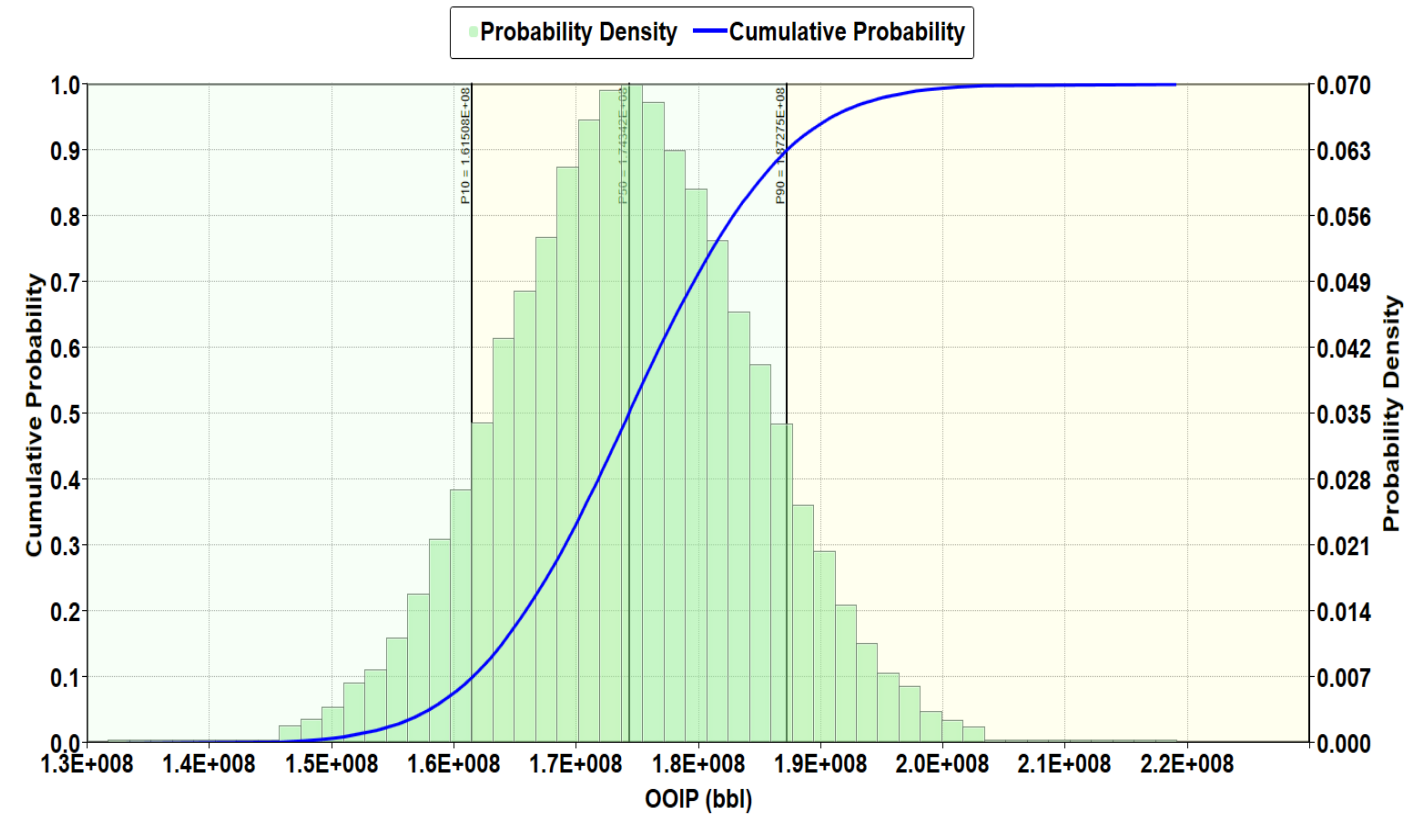

Figure A1: OOIP Probability Distribution Estimated by Constant Composition Initialized Reservoir Model 


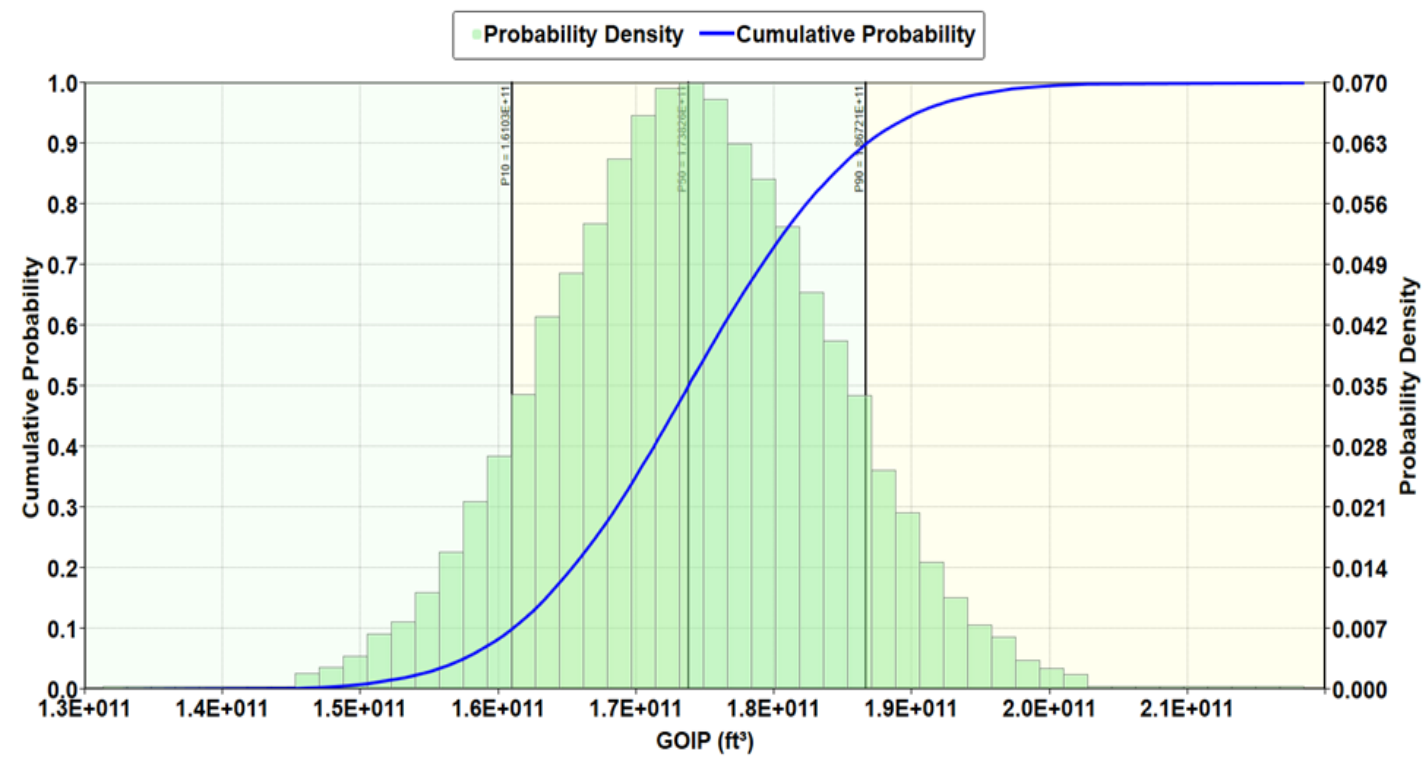

Figure A2: GOIP Probability Distribution Estimated by Constant Composition Initialized Reservoir Model

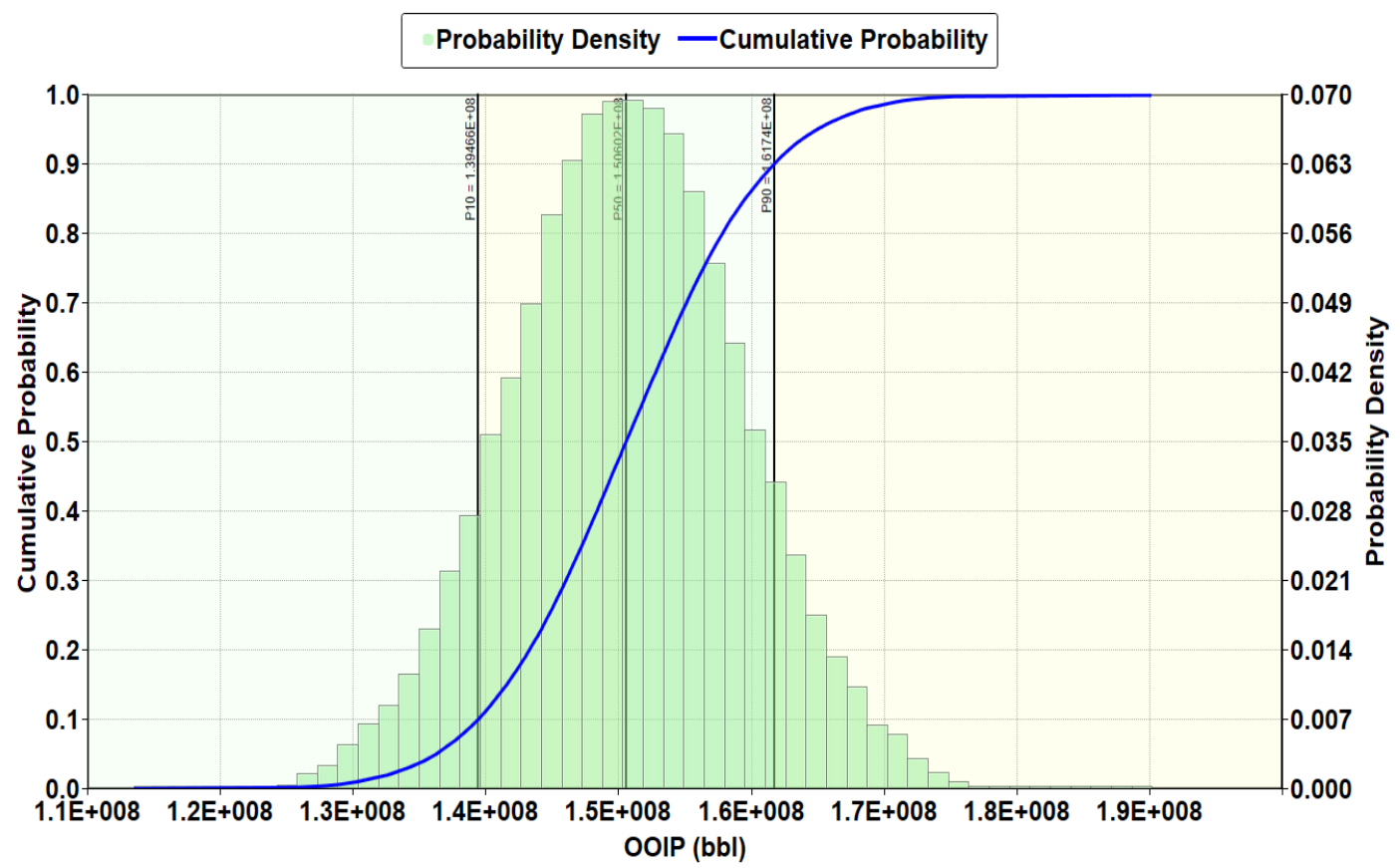

Figure A3: OOIP Probability Distribution Estimated by Isothermal CG Initialized Reservoir Model 


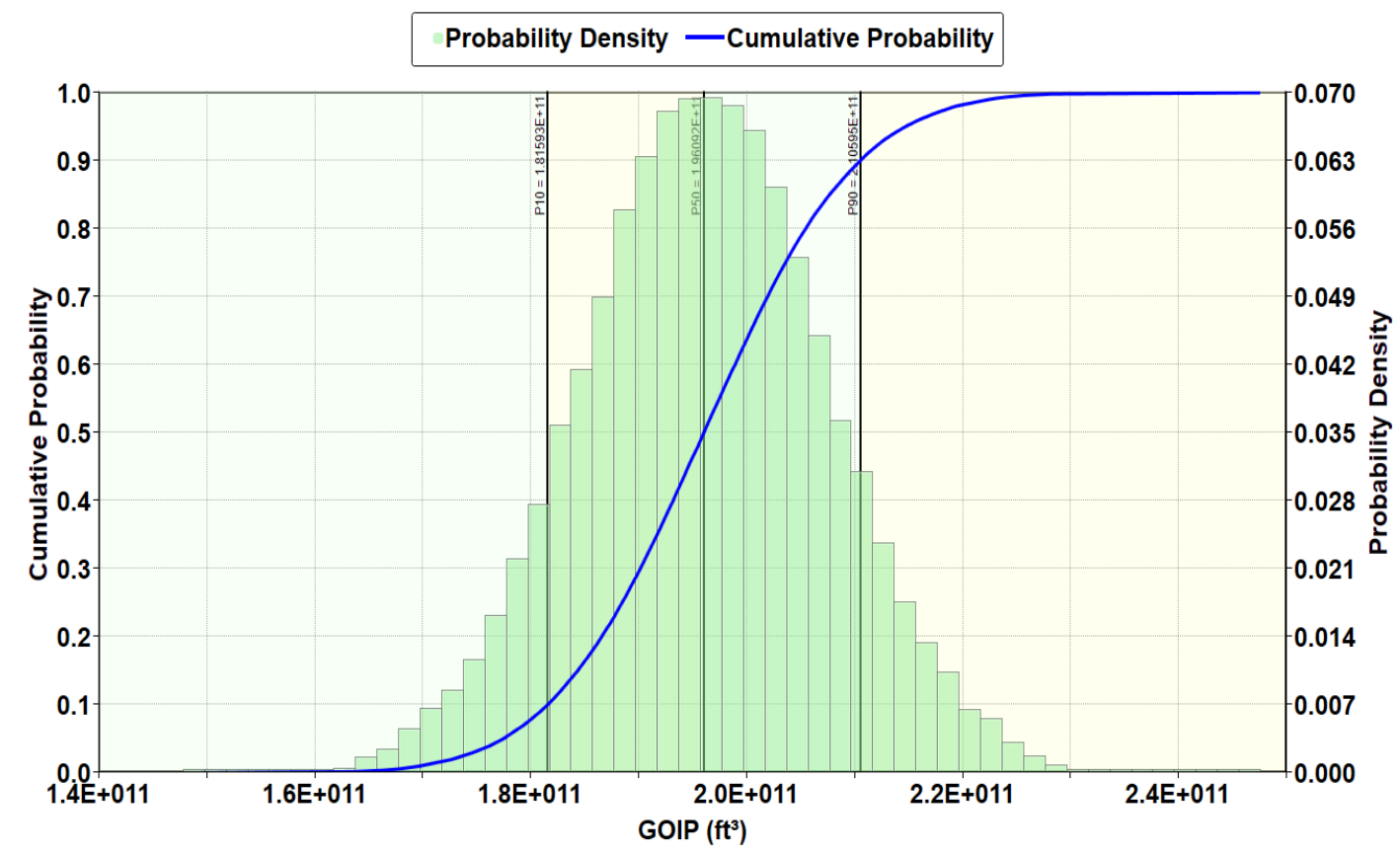

Figure A4: GOIP Probability Distribution Estimated by Isothermal CG Initialized Reservoir Model

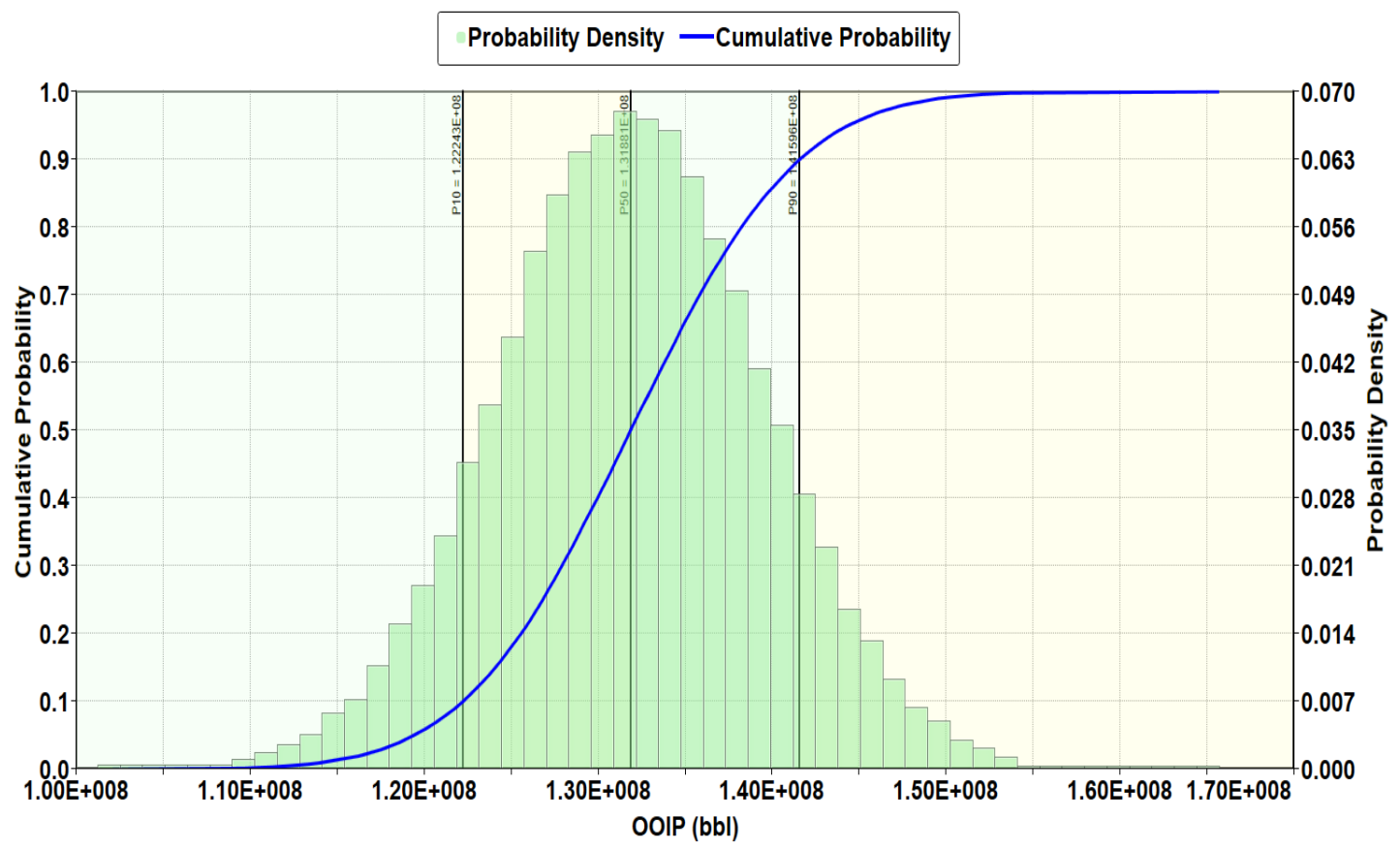

Figure A5: OOIP Probability Distribution Estimated by Zero Thermal Diffusion CG Initialized Reservoir Model 


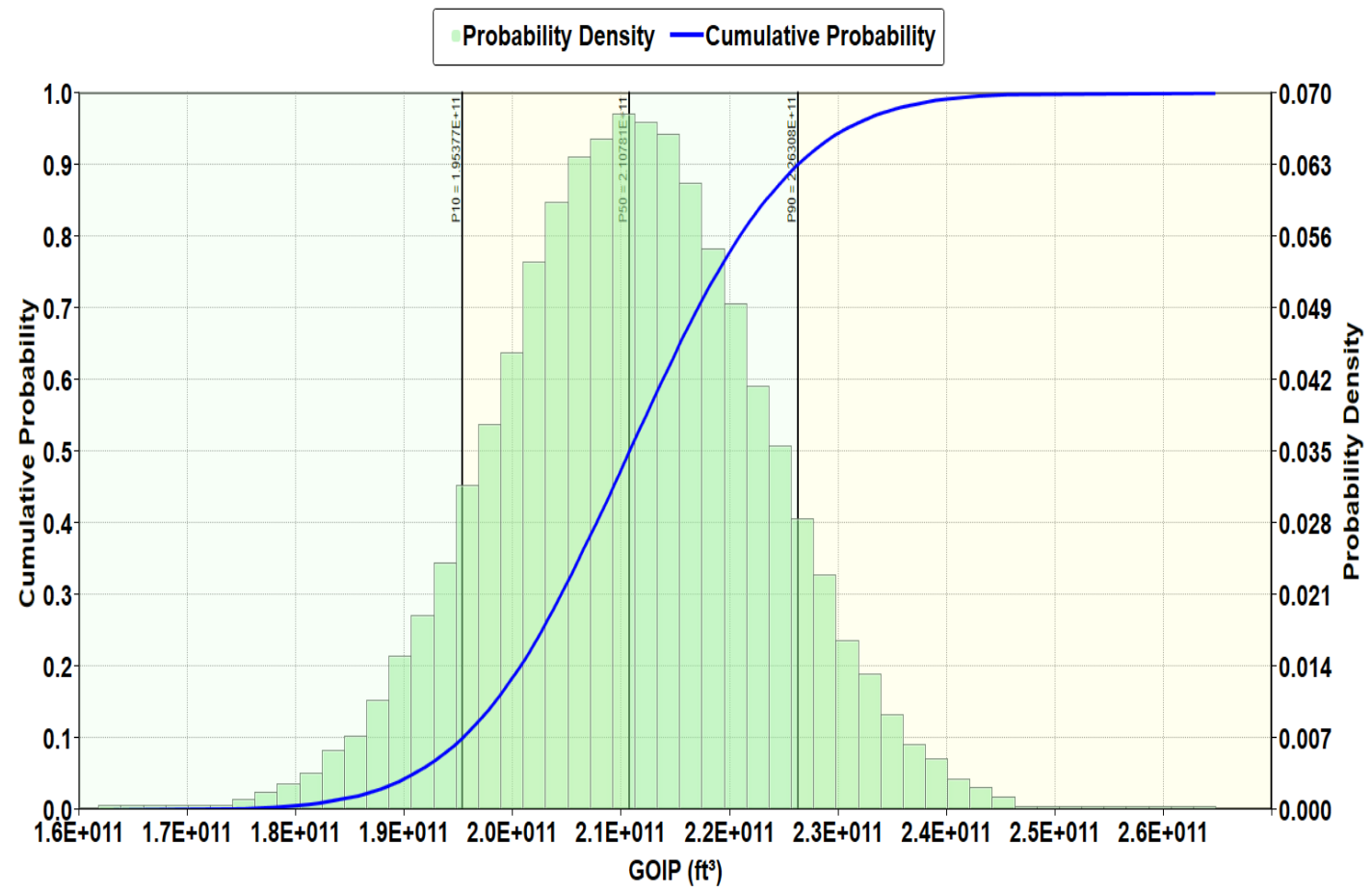

Figure A6: GOIP Probability Distribution Estimated by Zero Thermal Diffusion CG Initialized Reservoir Model

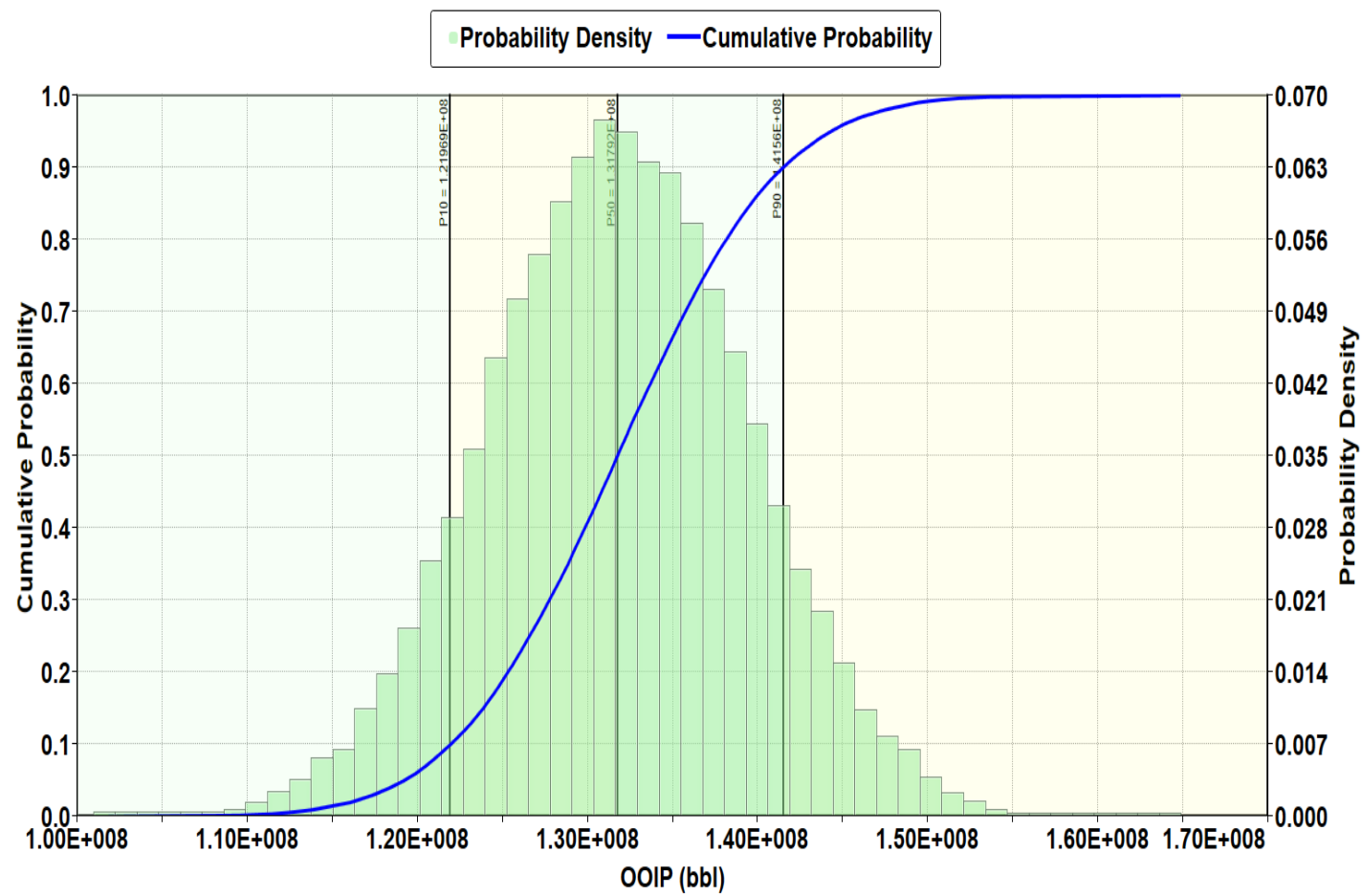

Figure A7: OOIP Probability Distribution Estimated by Haase's Thermal Diffusion CG Initialized Reservoir Model 


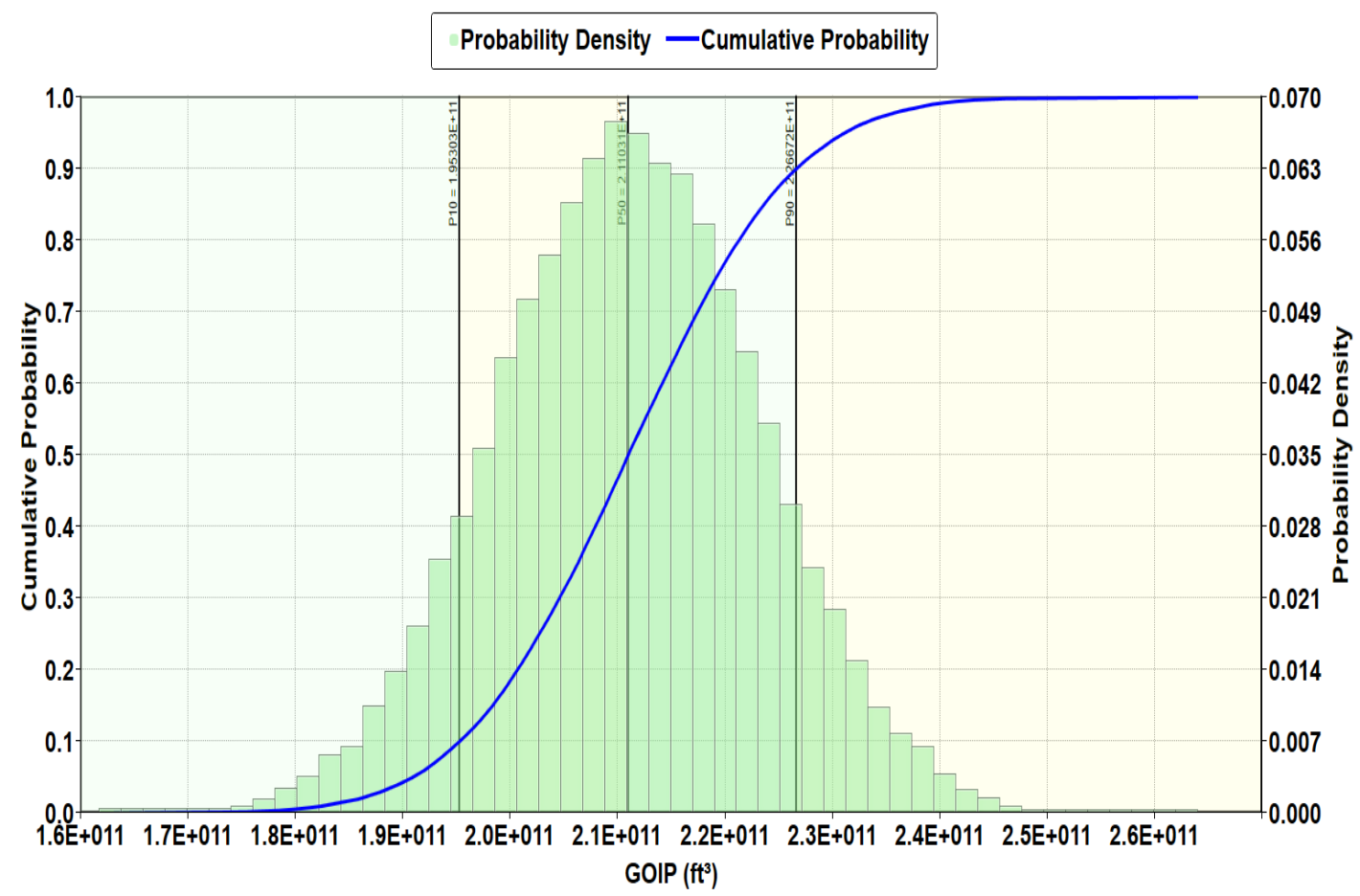

Figure A8: GOIP Probability Distribution Estimated by Haase's Thermal Diffusion CG Initialied Reservoir Model

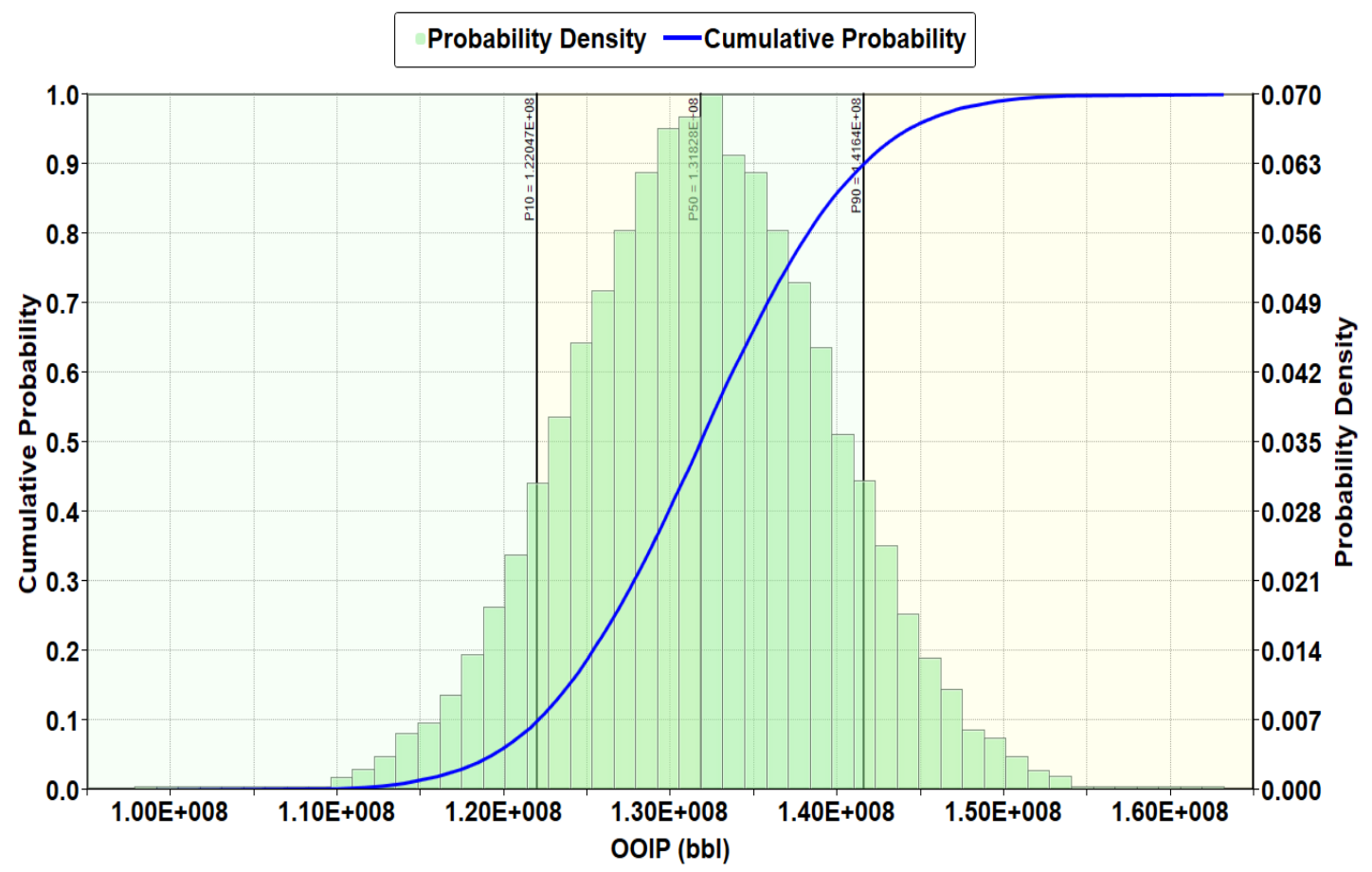

Figure A9: OOIP Probability Distribution Estimated by Kempers Thermal Diffusion CG Initialized Reservoir Model 


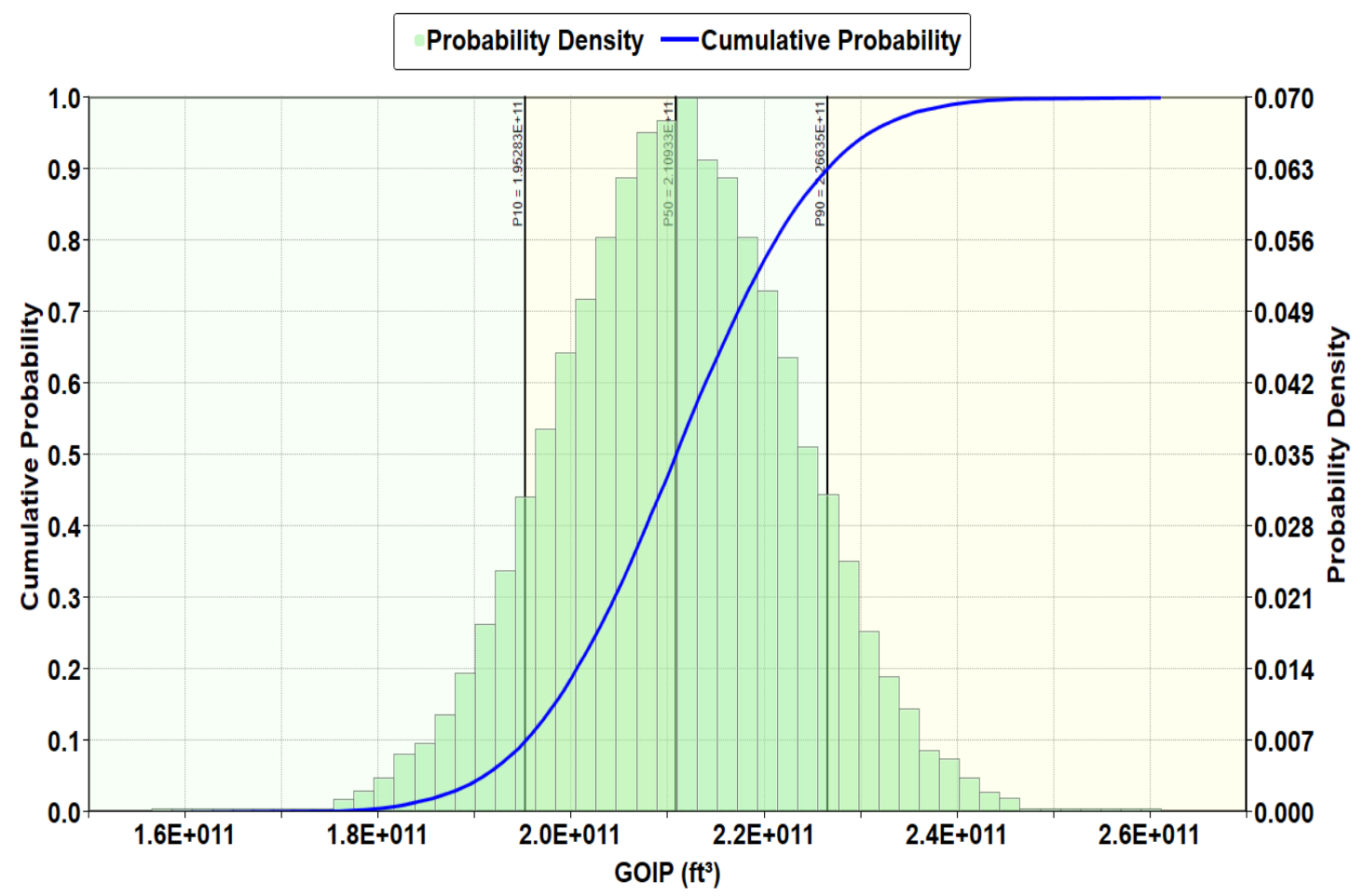

Figure A10: GOIP Probability Distribution Estimated by Kempers Thermal Diffusion CG Initialized Reservoir Model

- Response Surface Model Verification Plots for Oil Originally In-place (OOIP) and Gas Originally In-place (GOIP) for the Various Initialized Reservoir Models

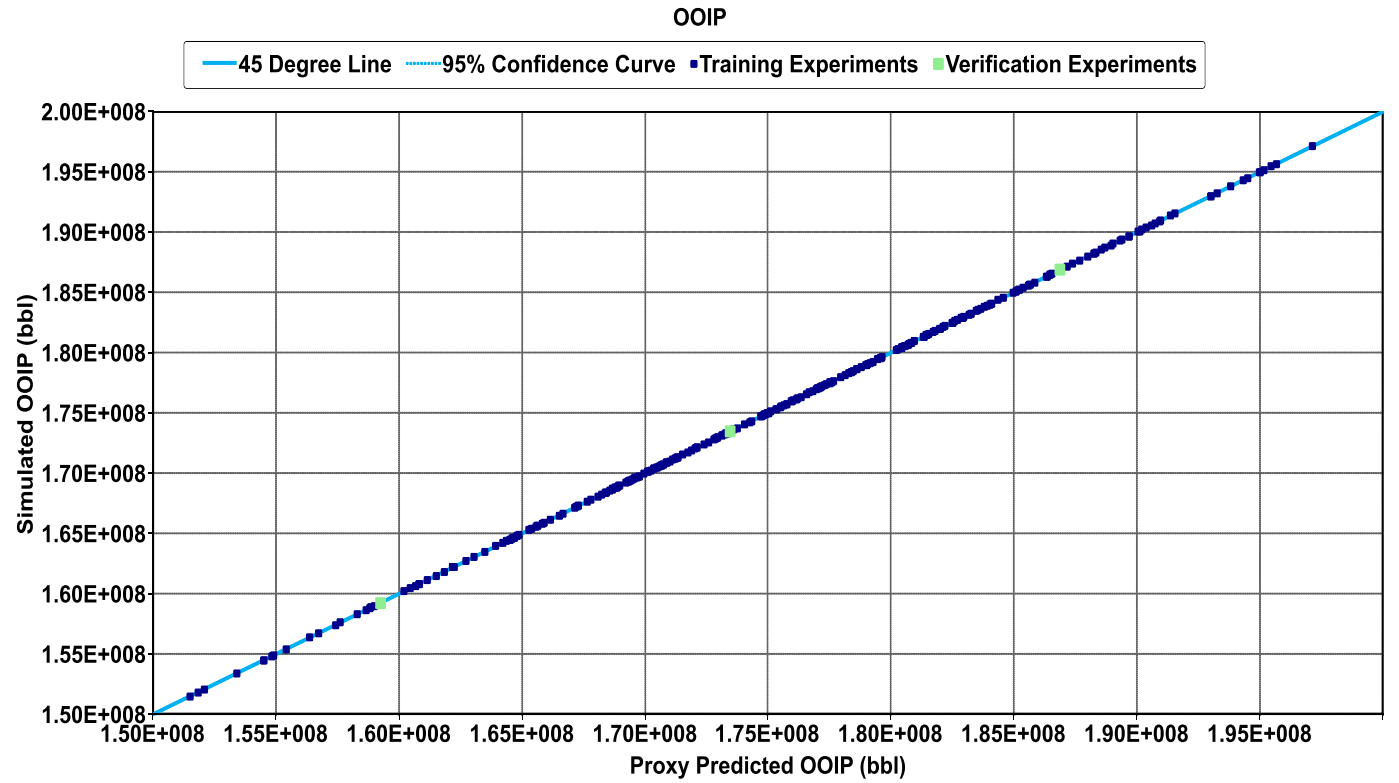

Figure B1: OOIP Response Surface Model Verification Plot for Constant Composition Initialized Reservoir Model 
GOIP

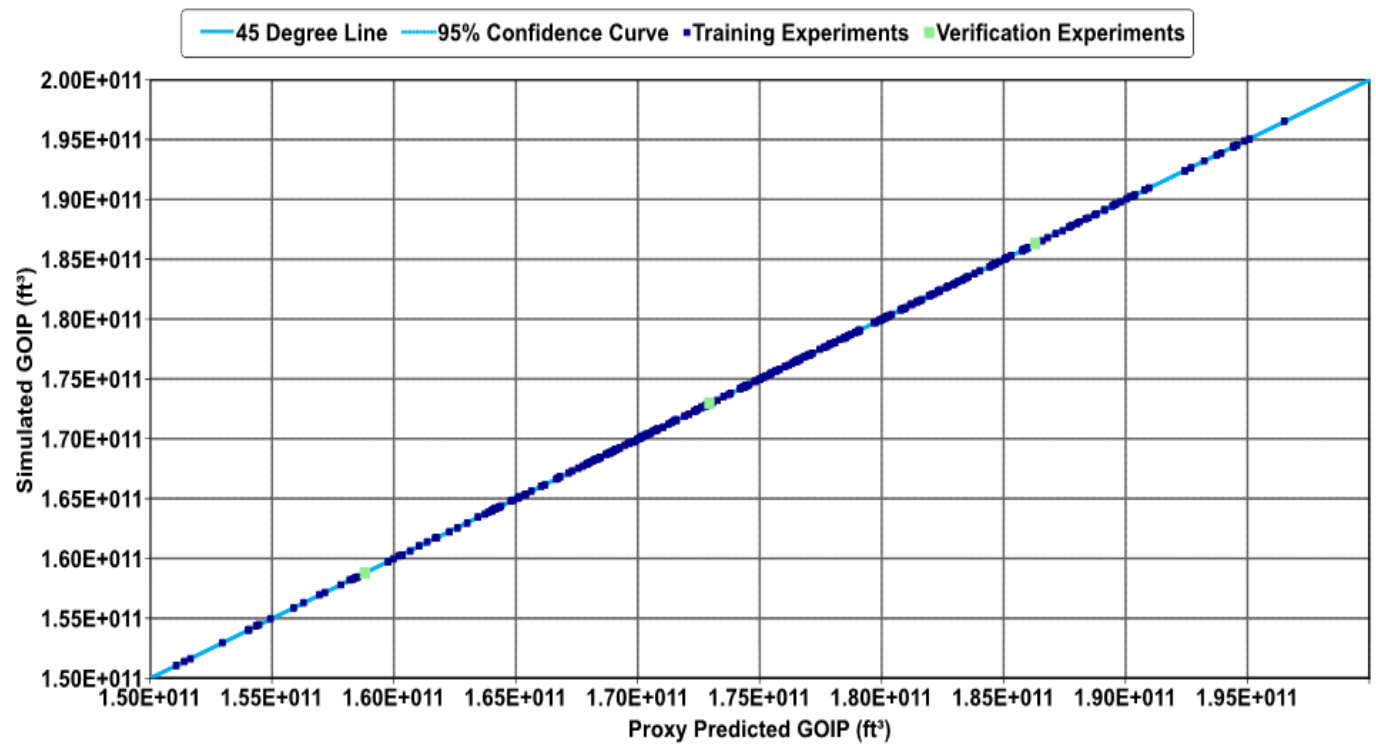

Figure B2: GOIP Response Surface Model Verification Plot for Constant Composition Initialized Reservoir Model

OOIP

- 45 Degree Line -...-95\% Confidence Curve -Training Experiments Verification Experiments

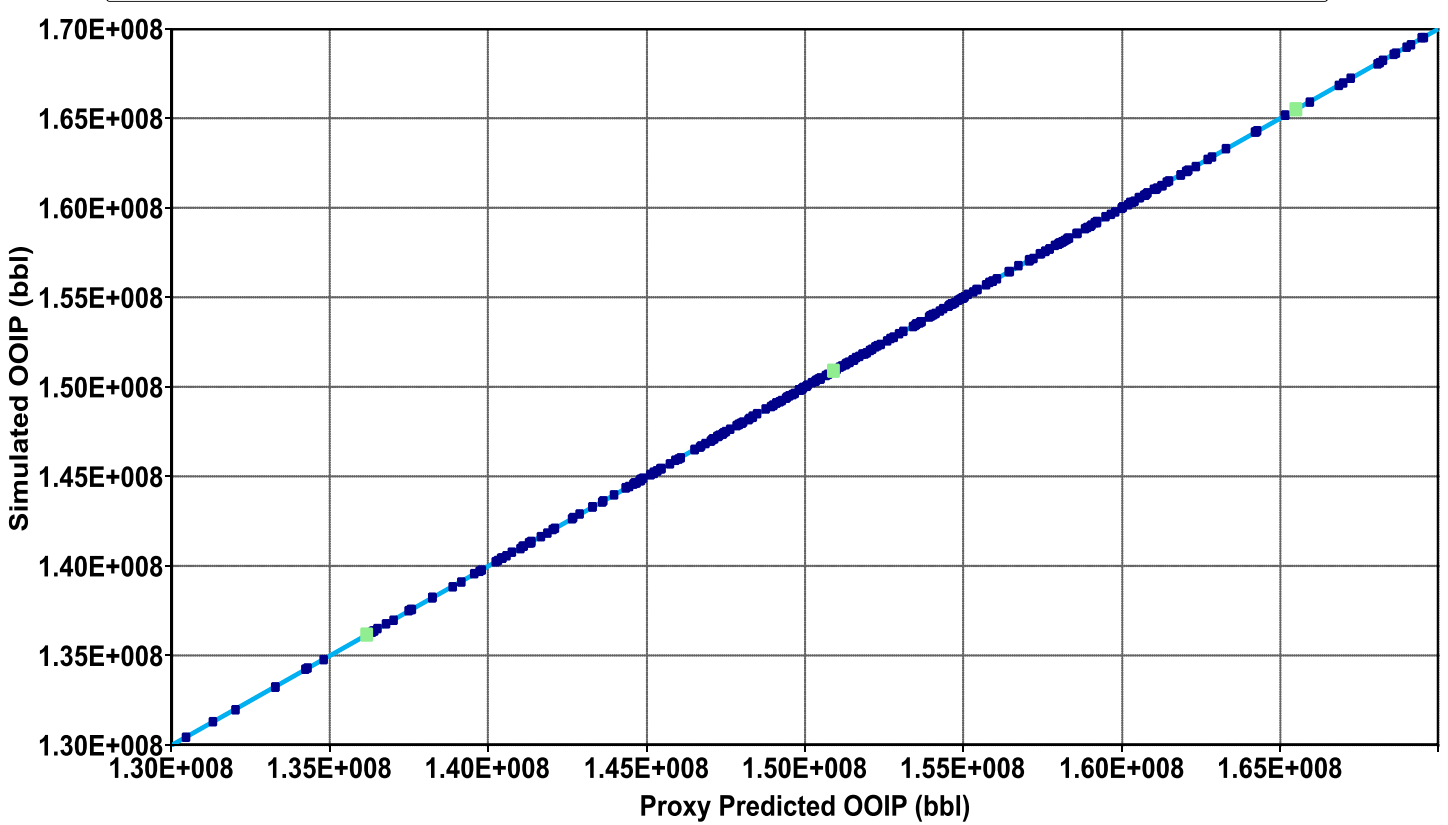

Figure B3: OOIP Response Surface Model Verification Plot for Isothermal CG Initialized Reservoir Model 
GOIP

- 45 Degree Line $\cdots \cdots \cdots \cdot 95 \%$ Confidence Curve -Training Experiments Verification Experiments

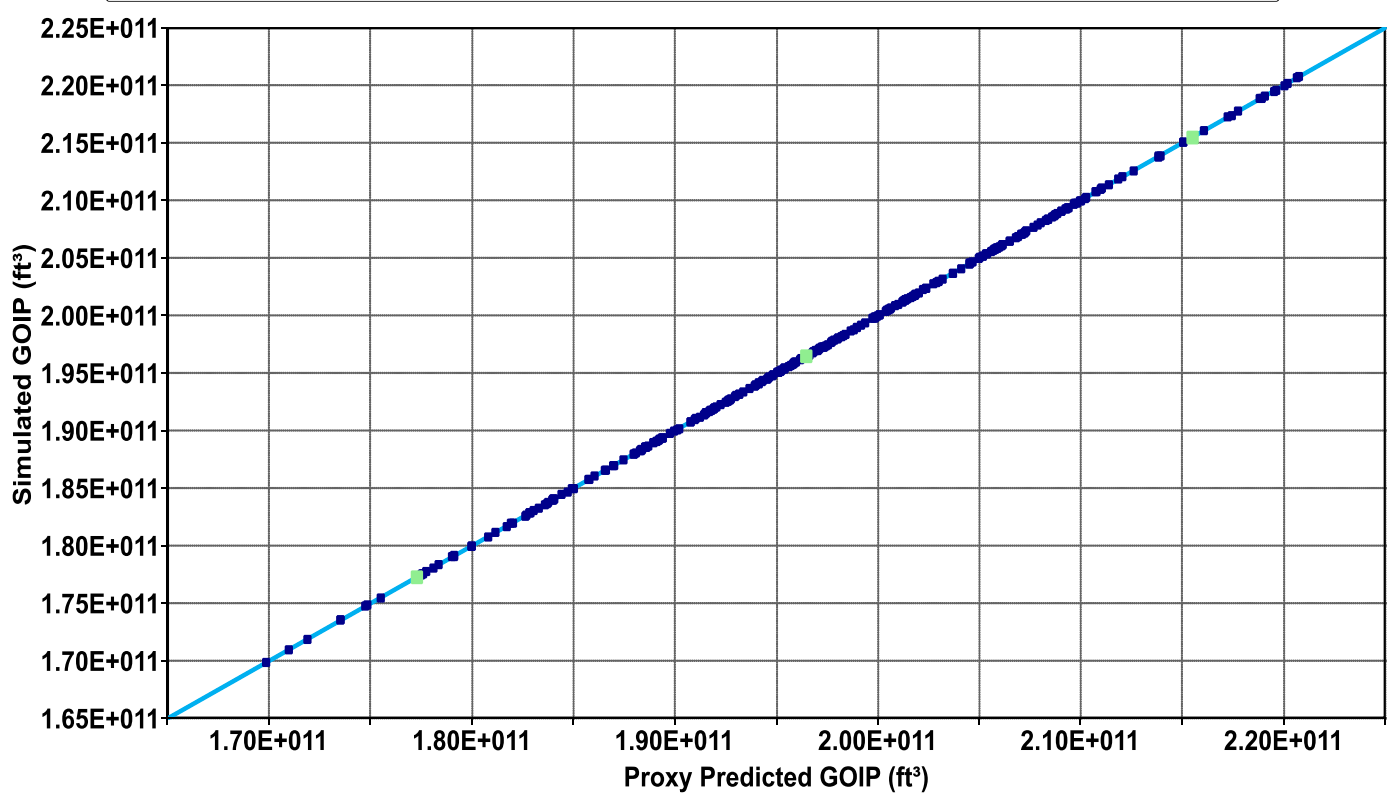

Figure B4: GOIP Response Surface Model Verification Plot for Isothermal CG Initialized Reservoir Model

OOIP

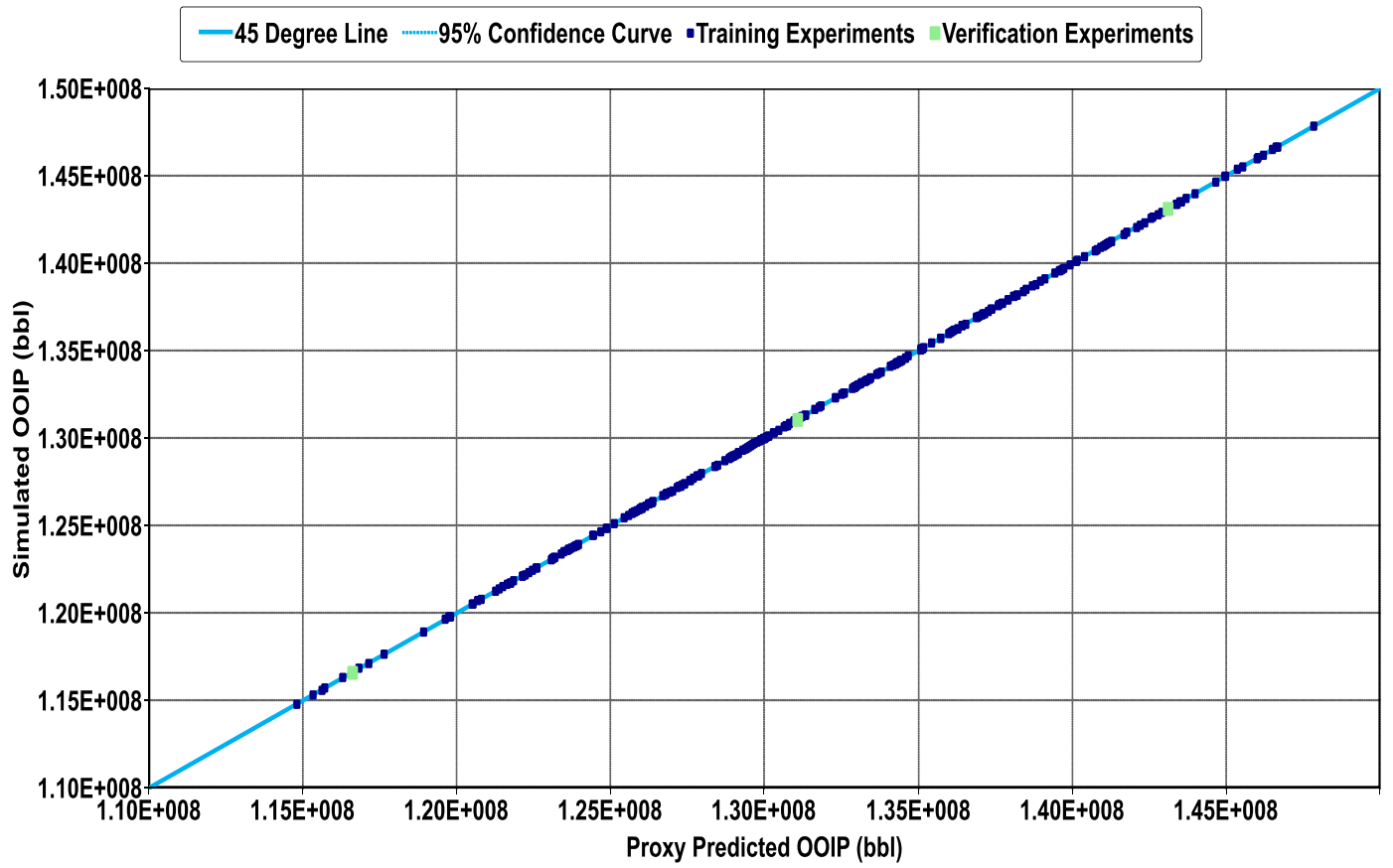

Figure B5: OOIP Response Surface Model Verification Plot for Zero Thermal Diffusion CG Initialized Reservoir Model 
GOIP

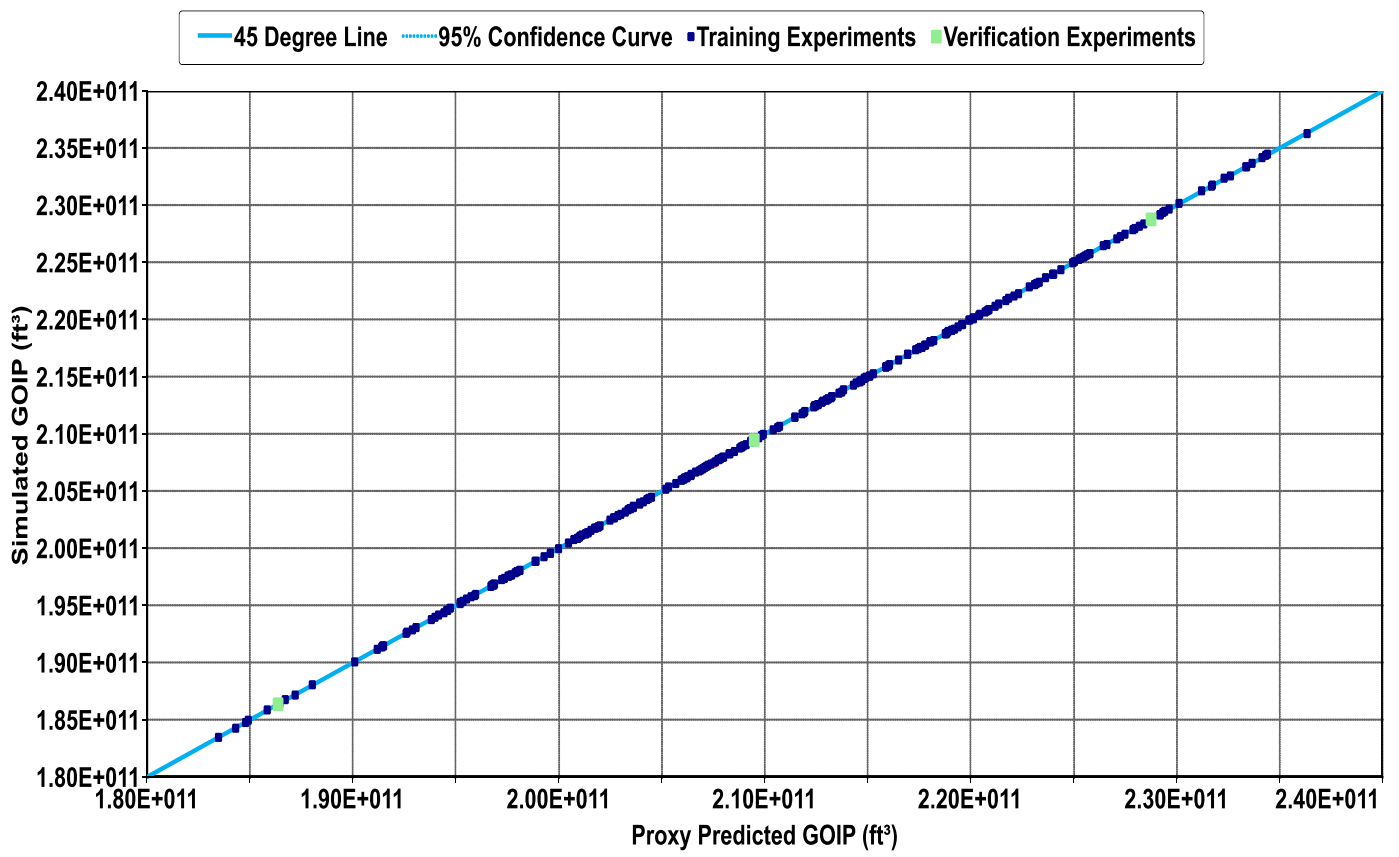

Figure B6: GOIP Response Surface Model Verification Plot for Zero Thermal Diffusion CG Initialized Reservoir Model

OOIP

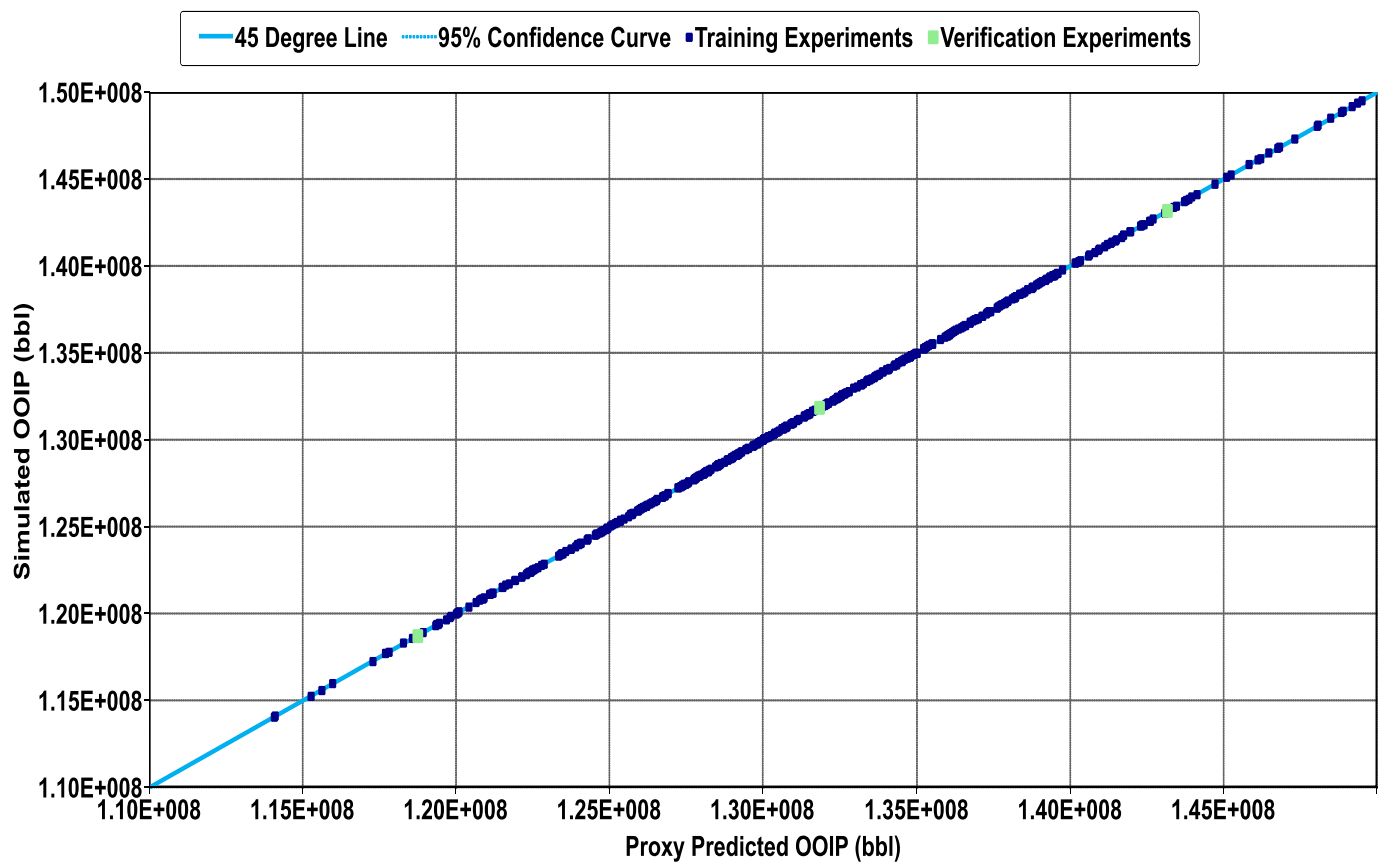

Figure B7: OOIP Response Surface Model Verification Plot for Haase's Thermal Diffusion CG Initialized Reservoir Mode 


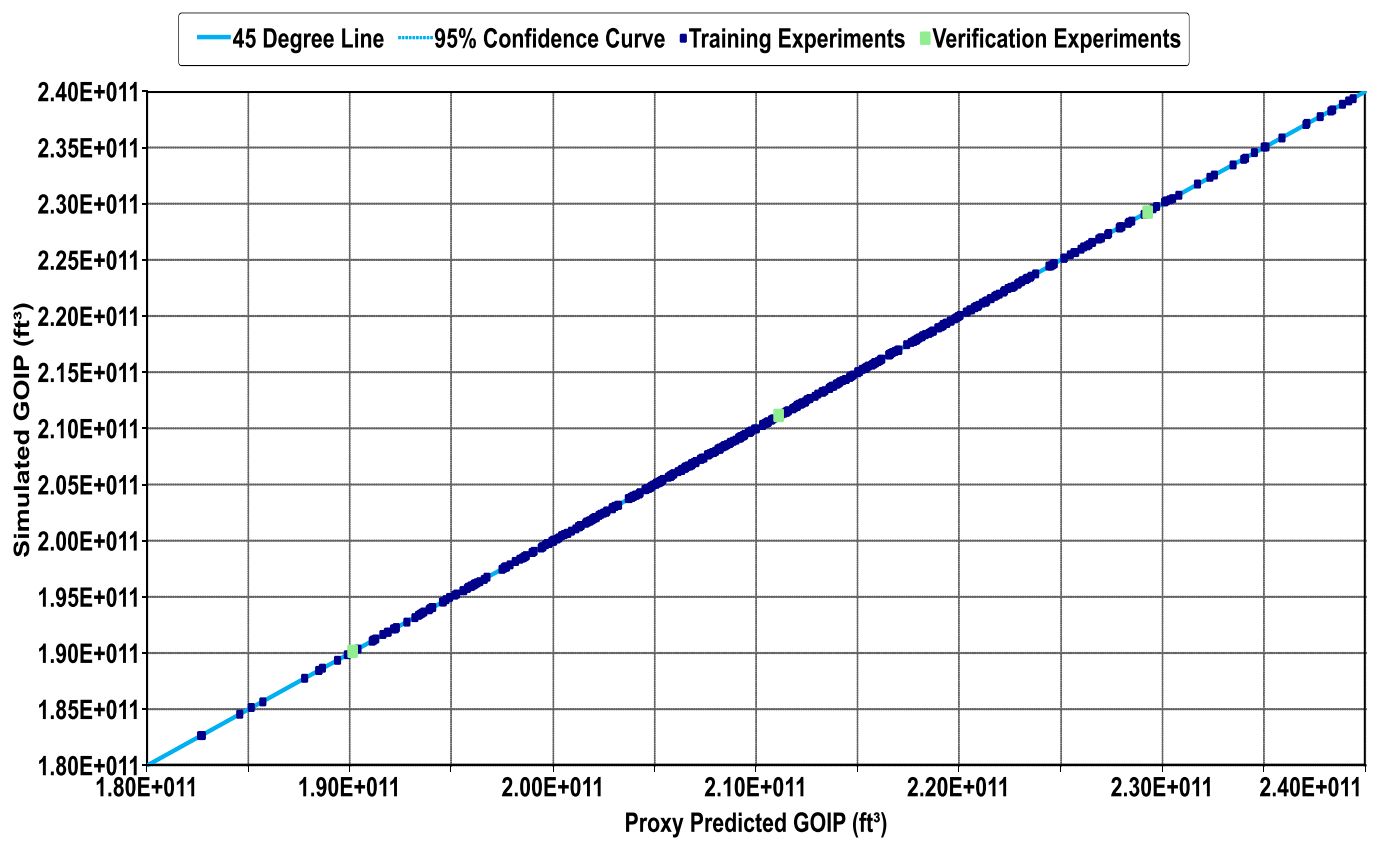

Figure B8: GOIP Response Surface Model Verification Plot for Haase's Thermal Diffusion CG Initialized Reservoir Model

OOIP

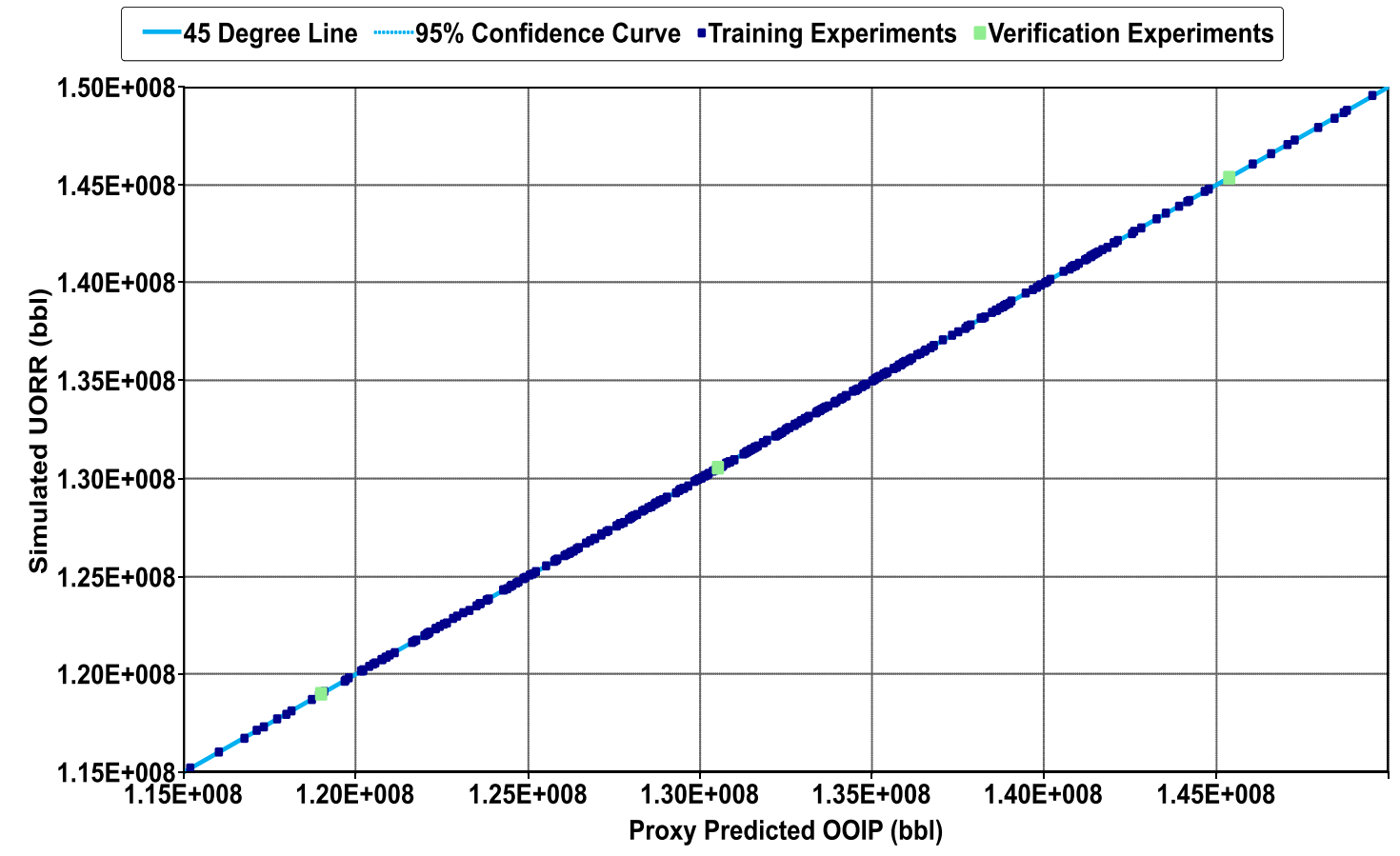

Figure B9: OOIP Response Surface Model Verification Plot for Kempers Thermal Diffusion CG Initialized Reservoir Model 
GOIP

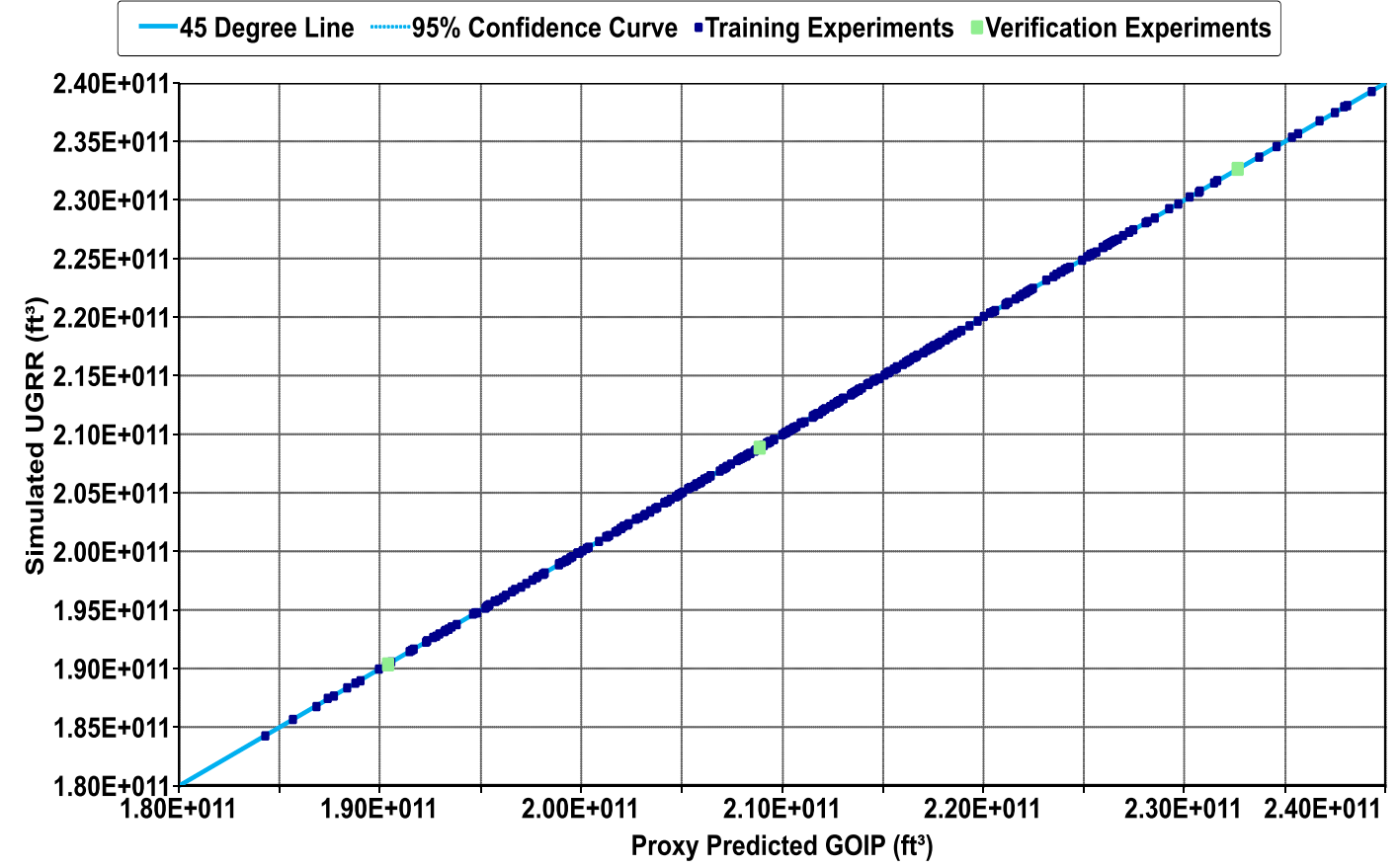

Figure B10: GOIP Response Surface Model Verification Plot for Kempers Thermal Diffusion CG Initialized Reservoir Model 